\title{
TRANSTROCHANTERIC ANTERIOR ROTATIONAL OSTEOTOMY FOR AVASCULAR NECROSIS OF THE FEMORAL HEAD
}

\author{
LONG-TERM RESULTS
}

MiCHAEL T. DEAN, MIGUEL E. CABANELA

From the Mayo Clinic and Mayo Foundation, Minnesota, USA

We reviewed 18 hips in 17 patients at a mean of five years after performing Sugioka's transtrochanteric anterior rotational osteotomy for avascular necrosis. The results were satisfactory in only three hips (17\%). Twelve hips had been revised by hip replacement, revision was pending in one and two others were unsatisfactory. Hip replacement was not compromised by the previous Sugioka osteotomy.

Fifteen hips (83\%) had shown further collapse of the femoral head, and we conclude from this and from isotope scans that the osteotomy may have impaired the residual blood supply of the femoral head. It seems that ethnic origin may be a factor in the outcome of this procedure; we have abandoned its use.

J Bone Joint Surg [ Br] 1993; 75-B:597-601.

Received 4 January 1993; Accepted 17 February 1993

The aims of treatment of avascular necrosis of the femoral head are to prevent collapse of the subchondral bone and to slow the progress of degenerative changes. These objectives are not reliably achieved by currently available operations. The natural history of avascular necrosis is not clear, but once collapse has occurred, joint destruction usually follows. Any procedure that would delay collapse would be useful.

Core decompression is of questionable value and is not indicated when necrosis has progressed beyond stage I or at most stage II of Ficat and Arlet (1980), and arthroplasty is reserved for advanced disease. Many different osteotomies have been advocated for the intermediate stages II and III. Sugioka (1978) reintroduced the concept of rotational osteotomy previously

M. T. Dean, MD, Senior Resident

Mayo Graduate School of Medicine, Rochester, Minnesota 55905, USA.

M. E. Cabanela, MD, Consultant

Department of Orthopedics, Mayo Clinic and Mayo Foundation, 200 First Street Southwest, Rochester, Minnesota 55905, USA.

Correspondence should be sent to Dr M. E. Cabanela.

(C) 1993 British Editorial Society of Bone and Joint Surgery $0301-620 \mathrm{X} / 93 / 4610 \$ 2.00$ described by Merle D'Aubigné et al (1965). The Sugioka osteotomy is at transtrochanteric level, and either anterior or posterior rotation of the head moves the affected area from the weight-bearing part of the joint, bringing unaffected joint surface into position for weight-bearing. In theory, the unaffected surface has normal cartilage and subchondral bone, and is able to prevent collapse of the femoral head.

In 1984, Sugioka reported excellent or good results in $86 \%$ of the hips of 274 patients at a follow-up of 2 to 11 years.

We have made a prospective study of this procedure since 1981, and now report our results.

\section{PATIENTS AND METHODS}

From 1981 to 1983, we performed transtrochanteric rotational osteotomy on 21 hips in 19 patients. Two patients were excluded from the study because of inadequate follow-up; 18 hips in 17 patients were therefore reviewed. They all had anterior rotational osteotomy with a mean rotation of $79^{\circ}\left(65^{\circ}\right.$ to $\left.95^{\circ}\right)$. The cause of avascular necrosis was alcohol abuse (6 hips), trauma (4), idiopathic (4), steroids (3) and Perthes' disease (one). The hips were graded for avascular necrosis using the stages of Ficat and Arlet (1980): 3 had stage IV, 12 had stage III, and 3 had stage II. No patient with hips in stage I was included.

All the osteotomies were performed by the same surgeon (MEC), after assessment by plain radiography, tomography, and ${ }^{99 \mathrm{~m} T c}$ bone scanning. The neck-shaft angles were measured before and after operation to record any valgus or varus change.

Surgical technique. Cephazolin $1 \mathrm{~g}$ was given to each patient. A lateral incision over the greater trochanter allowed the fascia lata to be split in line with the femur. The external rotator muscles were detached from the proximal end of the greater trochanter, which was osteotomised to allow it and the attached abductor muscles to be retracted proximally.

A circumferential capsular incision was made at the level of the labrum. Under radiographic control, two 
Table I. Details and results of 19 patients treated by transtrochanteric anterior rotational osteotomy for avascular necrosis of the hip

\begin{tabular}{|c|c|c|c|c|c|c|c|c|c|c|c|c|c|c|}
\hline \multirow[b]{2}{*}{ Case } & \multirow[b]{2}{*}{ Side } & \multirow[b]{2}{*}{$\begin{array}{l}\text { Age } \\
\text { (yr) }\end{array}$} & \multirow[b]{2}{*}{$\begin{array}{l}\text { Ficat } \\
\text { stage }\end{array}$} & \multirow[b]{2}{*}{ Cause } & \multirow{2}{*}{$\begin{array}{l}\text { Rotational } \\
\text { correction } \\
\text { (degrees) }\end{array}$} & \multirow[b]{2}{*}{$\begin{array}{l}\text { Follow-up } \\
\text { (mth) }\end{array}$} & \multicolumn{4}{|l|}{ Result } & \multicolumn{2}{|c|}{$\begin{array}{l}\text { Collapse of } \\
\text { femoral head }\end{array}$} & \multirow[b]{2}{*}{$\begin{array}{l}\text { Overall } \\
\text { result }\end{array}$} & \multirow{2}{*}{$\begin{array}{l}\text { Revision to } \\
\text { total hip } \\
\text { arthroplasty }\end{array}$} \\
\hline & & & & & & & Pain & $\begin{array}{l}\text { Range of } \\
\text { motion }\end{array}$ & Limp & $\begin{array}{l}\text { Joint } \\
\text { narrowing }\end{array}$ & Before & After & & \\
\hline 1 & Right & 44 & IV & Alcoholism & 70 & 48 & Moderate & Good & Severe & Yes & Yes & Yes & Poor & Yes \\
\hline 2 & Left & 41 & IV & Idiopathic & 90 & 101 & Severe & Poor & Severe & Yes & Yes & Yes & Poor & Pending \\
\hline 3 & Left & 26 & III & Steroids & 70 & 52 & Severe & Poor & Severe & Yes & No & Yes & Poor & Yes \\
\hline 4 & Left & 17 & II & Traumatic & 80 & 106 & Mild & Poor & Mild & Yes & No & Yes & Good & No \\
\hline 5 & Right & 37 & II & Idiopathic & 75 & 62 & Moderate & Good & Moderate & No & No & Yes & Poor & No \\
\hline 6 & Right & 24 & III & Idiopathic & 80 & 19 & Severe & Good & Severe & Yes & No & Yes & Poor & Yes \\
\hline 7 & Right & 49 & III & Alcoholism & 75 & 68 & Moderate & Good & Severe & Yes & No & Yes & Poor & Yes \\
\hline 8 & Left & 22 & IV & $\begin{array}{l}\text { Perthes' } \\
\text { disease }\end{array}$ & 90 & 30 & Severe & Poor & Severe & Yes & Yes & Yes & Poor & Yes \\
\hline 9 & Right & 38 & III & Idiopathic & 70 & 55 & Moderate & Good & Slight & Yes & No & Yes & Poor & Yes \\
\hline \multirow[t]{2}{*}{10} & Right & 33 & III & Alcoholism & - & 27 & Moderate & Poor & Slight & No & No & Yes & Poor & Yes \\
\hline & Left & & III & - & 65 & 30 & Severe & Poor & Severe & Yes & No & Yes & Poor & Yes \\
\hline 11 & Right & 49 & III & Alcoholism & 85 & 24 & Severe & Poor & Severe & Yes & No & Yes & Poor & Yes \\
\hline 12 & Right & 36 & II & Steroids & 75 & 60 & Severe & Poor & Severe & Yes & No & Yes & Poor & Yes \\
\hline 13 & Right & 30 & III & Steroids & 80 & 96 & None & Good & None & Yes & No & Yes & Good & No \\
\hline 14 & Left & 57 & III & Trauma & 75 & 60 & None & Good & Mild & No & No & No & Good & No \\
\hline 15 & Left & 46 & III & Alcoholism & 75 & 60 & Moderate & Good & Severe & No & No & No & Poor & No \\
\hline 16 & Right & 23 & III & Trauma & 95 & 18 & Severe & Poor & Severe & No & No & No & Poor & Yes \\
\hline 17 & Right & 29 & III & Trauma & 90 & 44 & Severe & Good & Severe & Yes & No & Yes & Poor & Yes \\
\hline \multirow[t]{2}{*}{$18^{*}$} & Right & 32 & III & Steroids & 90 & 12 & - & - & - & - & - & - & - & - \\
\hline & Left & & III & - & 90 & 6 & - & - & - & - & - & - & - & - \\
\hline $19^{*}$ & Right & 42 & III & Alcoholism & 85 & 3 & - & - & - & - & - & - & - & - \\
\hline
\end{tabular}

* lost to follow-up

Kirschner wires were driven through the centre of the cut surface of the femur perpendicular to its neck and aimed at the lesser trochanter. Double osteotomy was then performed using a reciprocating saw: the first cut was in the plane of the Kirschner wires and the second was made on the medial side of the femur at the level of the lesser trochanter, perpendicular to the first, as described by Sugioka (1978).

The proximal fragment was then rotated anteriorly, about the axis of the femoral neck, so that the involved portion of the femoral head was removed from the weight-bearing surface. This position of rotation was held temporarily by a Kirschner wire, and the osteotomy was fixed by a Richards sliding screw device with a side plate. In addition, a $6.5 \mathrm{~mm}$ cancellous screw was placed across the osteotomy to control rotation. Compression was then applied to the sliding screw to achieve solid fixation. The wires used to align the osteotomy were removed, the capsule was repaired, and the trochanter reattached by 18-gauge wire. The wound was then thoroughly irrigated with sterile saline and two deep drains were placed in position. The fascia lata was approximated with absorbable sutures and the skin and subcutaneous tissues closed.

Three days after the operation, patients were allowed to walk with crutches, touch weight-bearing on the affected side, and they continued to do so after leaving hospital until there was radiographic evidence of solid union.

Follow-up averaged 57 months (18 to 106). In patients who did not require arthroplasty, it averaged 81 months; in those who required arthroplasty it was taken to be the interval between osteotomy and the subsequent operation.

For 15 patients follow-up included clinical examination performed by the senior author (MEC) and radiography. The other two patients had had hip replacements at other institutions, and were reported by them.

The grading of clinical results was based on pain, 


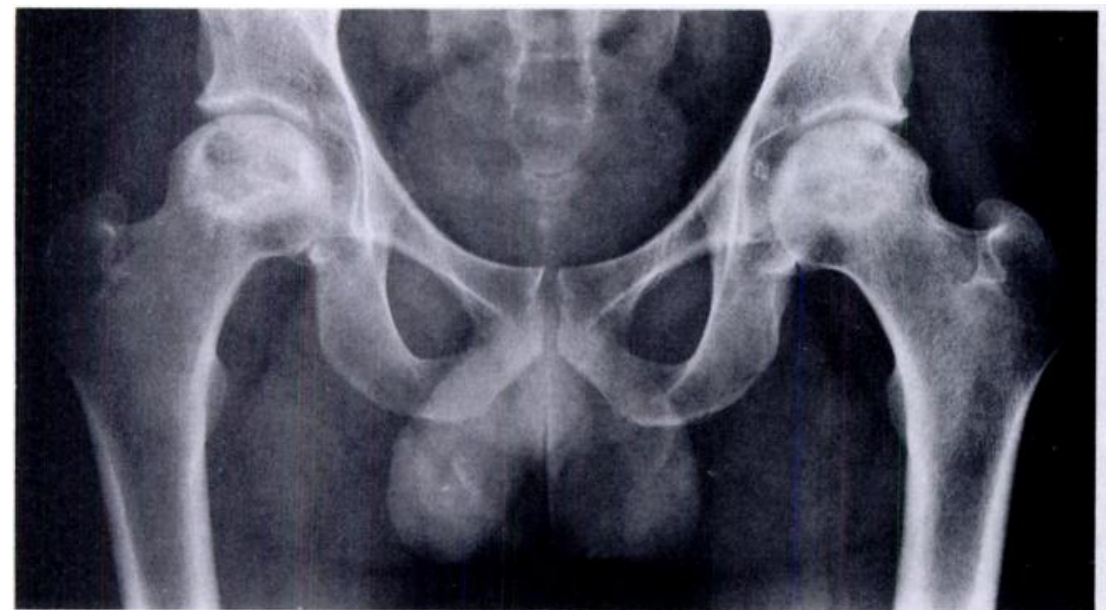

Fig. 1a

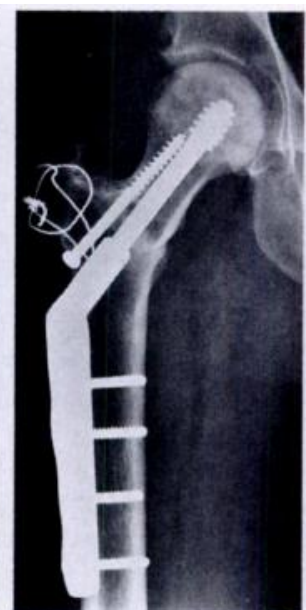

Fig. 1b

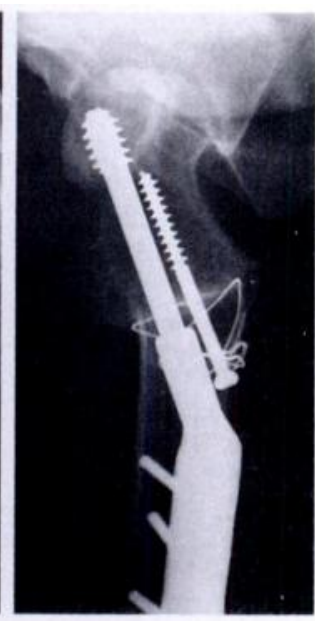

Fig. 1c

Case 10. Figure la - Radiograph of a 33-year-old man with bilateral, alcohol-induced, stage III avascular necrosis of the femoral heads. Figure $1 \mathrm{~b}$ - Anteroposterior view of right hip six months after transtrochanteric anterior rotational osteotomy. The early result was satisfactory. Figure lc - Lateral view.

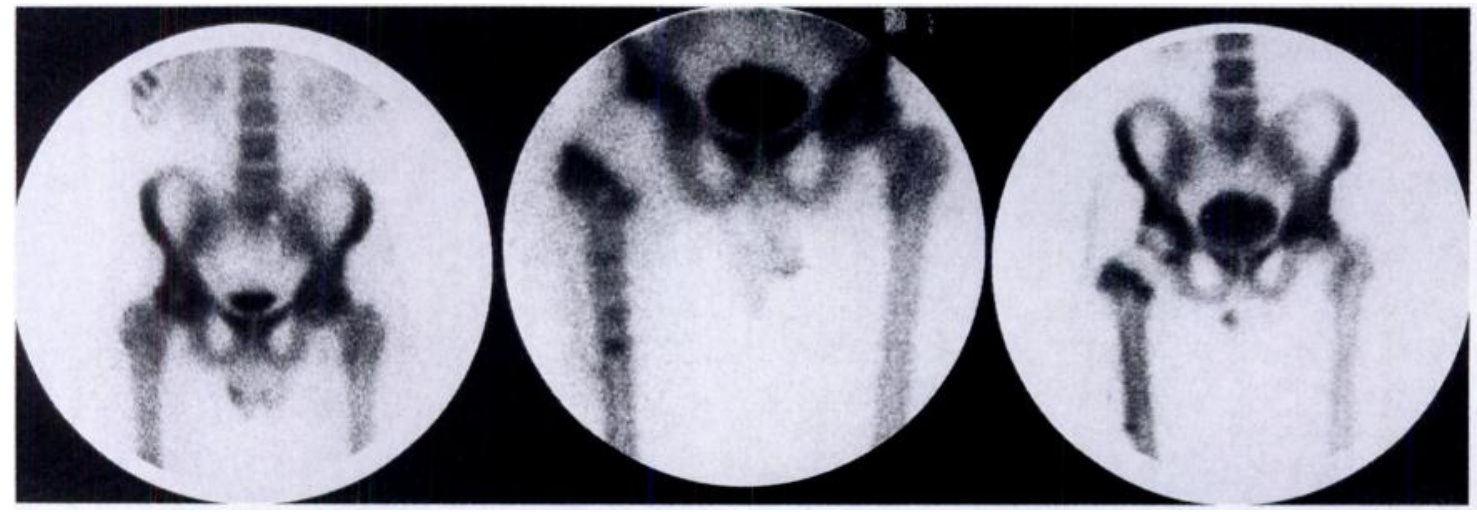

Fig. 2

Case 10. Left, ${ }^{99 \mathrm{~m}} \mathrm{Tc}$ bone scan before operation. Middle, two weeks after operation. Right, six months after operation. There is a reduction in isotope uptake in the head and neck of the femur.

limp (none, mild, moderate, or severe), and range of motion (excellent, good, or poor).

\section{RESULTS}

For all 21 hips, total blood loss averaged $600 \mathrm{ml}$ and the average operating time was 182 minutes. Hospital stay ranged from 8 to 14 days. The average time to union of the osteotomy was 10 weeks.

At review, 12 of the 18 hips had required total arthroplasty for continued pain, or progression of symptoms (Table I). The mean time to revision for these 12 hips was 39 months, by which time all showed further narrowing of the joint space or progression of femoral head collapse (Figs 1 to 4). The previous Sugioka osteotomy did not cause problems at revision arthroplasty.

One other patient has a poor clinical and radiographic result and is awaiting total hip replacement. At their latest follow-up two other patients had moderate to severe pain and radiological evidence of collapse of the femoral head and degenerative changes.

This leaves only three patients with satisfactory results. The first was a 17-year-old boy with stage II posttraumatic avascular necrosis. Nine years after osteotomy he has only mild pain and a slight limp, but the range of motion is limited and there is radiographic joint narrowing.

The second patient, a 57-year-old woman with posttraumatic avascular necrosis in stage III, has an excellent clinical and radiographic result at five years. She has good range of motion, no pain, and only a mild limp with no evidence of joint-space narrowing or further collapse (Figs 5 to 8). The third patient, a 30-year-old man with steroid-induced avascular necrosis in stage III, has no pain or limp eight years after osteotomy. His range of hip motion is good, but radiographs show some further collapse of the femoral head and joint-space narrowing.

One of three patients with stage II disease had a satisfactory result; 10 of 11 patients (12 hips) with stage III disease and all 3 with stage IV had poor results. The ${ }^{99 \mathrm{~m}} \mathrm{Tc}$ bone scans of all patients showed additional and 


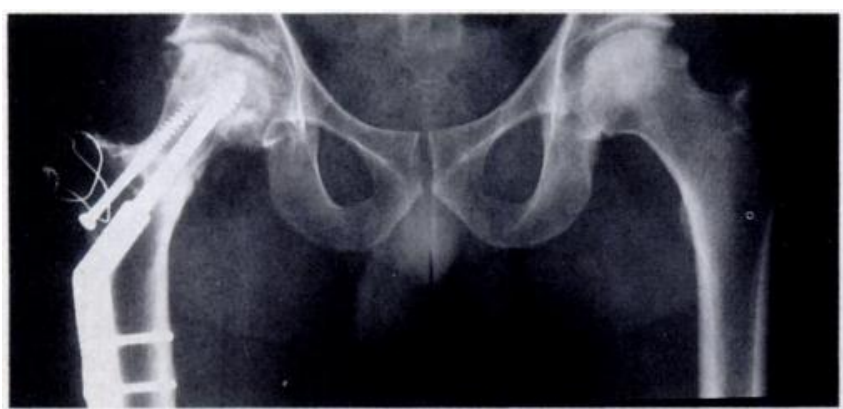

Fig. 3

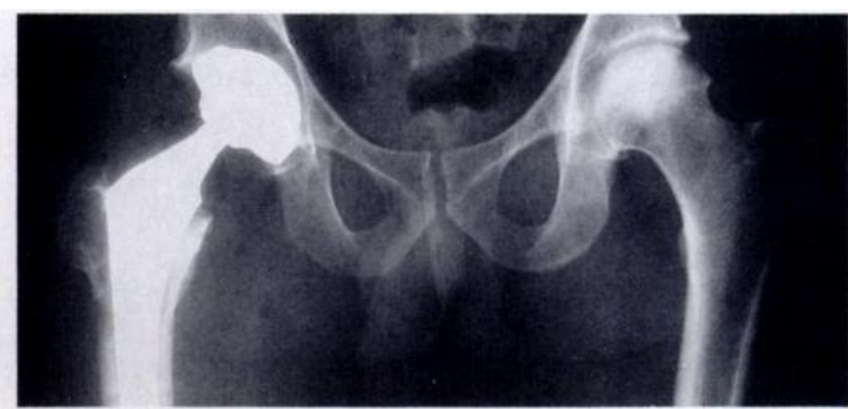

Fig. 4

Case 10. Figure 3 - 3.5 years after operation. There are degenerative changes and collapse of the femoral head. Figure 4 - Conversion to an uncemented hip replacement arthroplasty gave a satisfactory outcome.

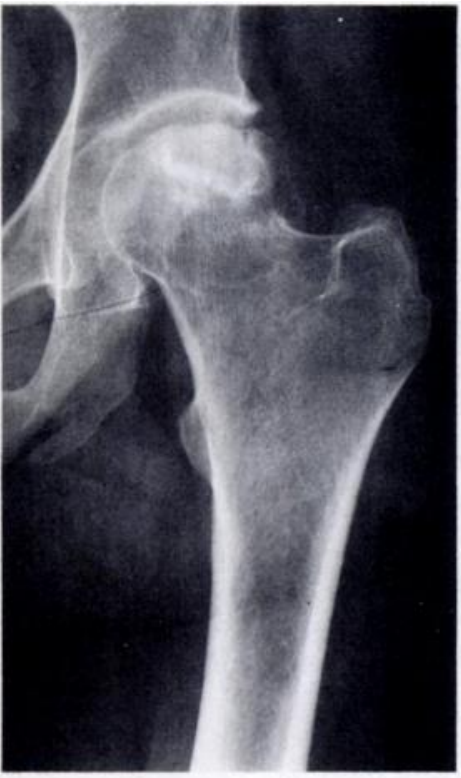

Fig. 5a

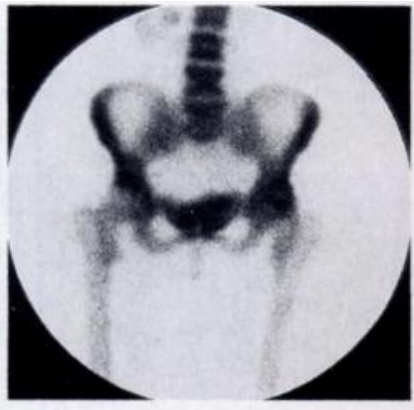

Fig. 5b

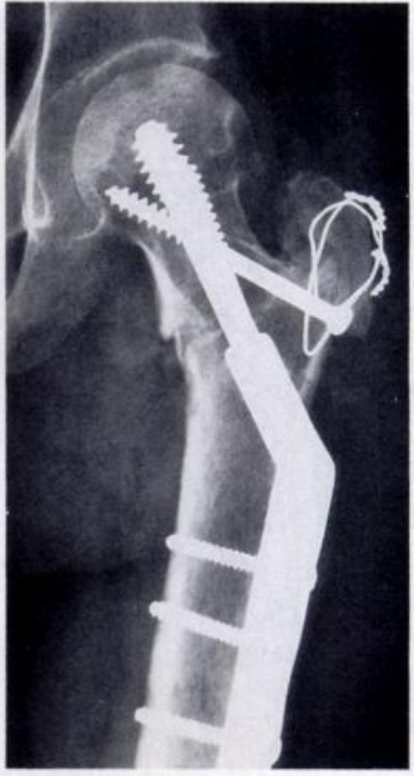

Fig. 6a

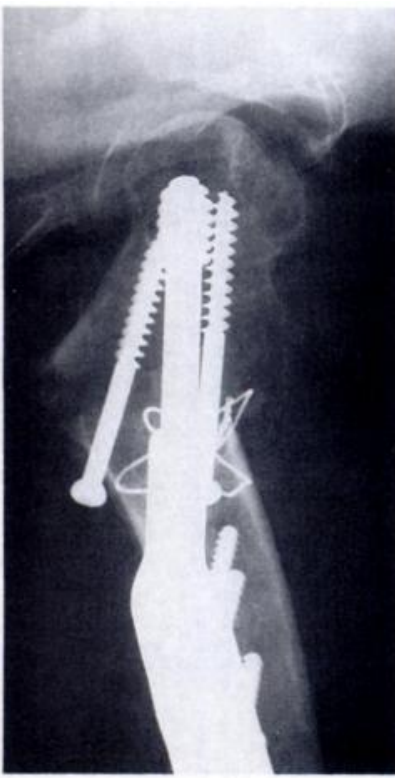

Fig. 6b

Case 14. Figure 5a - Post-traumatic stage III avascular necrosis of the femoral head in a 57-year-old woman. Figure $5 \mathrm{~b}-{ }^{99 \mathrm{~m}} \mathrm{Tc}$ bone scan before operation. Figure 6 - Anteroposterior (a) and lateral (b) views one month after operation. The anterior translation of the necrotic area can be seen.

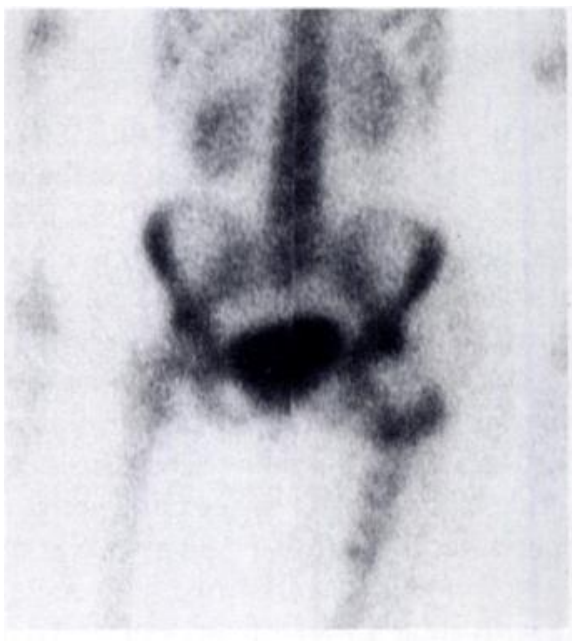

Fig. 7

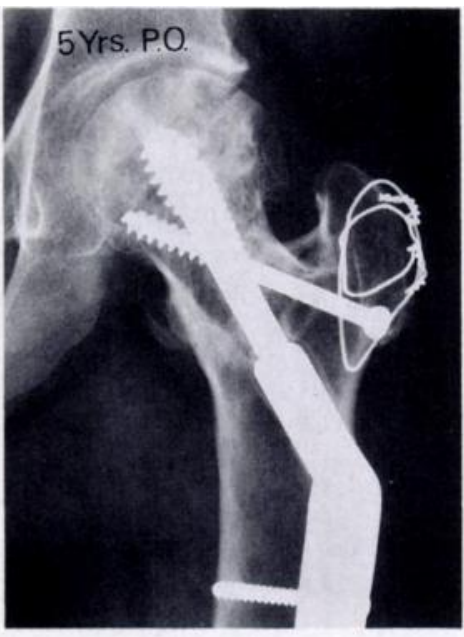

Fig. 8
Figure 7 - $99 \mathrm{~m} \mathrm{Tc}$ bone scan three months after the operation showing reduced uptake at the head and neck of the femur. Figure 8 - Anteroposterior view of the hip five years after operation. The patient had a satisfactory result with few symptoms. 
persistent impairment of the vascular supply to the femoral head soon after the operation (Fig. 2).

The neck-shaft angles averaged $133^{\circ}$ before and $142^{\circ}$ after operation. The angle increased in 18 hips (valgus) and decreased in three (varus), but the achievement of a varus neck-shaft angle did not correlate with a better result.

In summary, of 18 hips, only three $(17 \%)$ had a satisfactory clinical result and two of these three showed ominous radiological signs.

There were no postoperative complications of clinical significance. One patient had respiratory distress soon after surgery, but this responded to treatment. There were no postoperative infections and there were no cases of malunion or nonunion. At follow-up, 10 of the $18 \mathrm{had}$ loss of joint motion at some stage, there was radiological evidence of progressive collapse in 13, and 11 showed increased joint-space narrowing.

\section{DISCUSSION}

In the last published study of the procedure Sugioka, Katsuki and Hotokebuchi (1982) reported $86 \%$ good or excellent results in 274 patients at follow-up of 2 to 11 years, and in 1991 Sugioka, Hotokebuchi and Tsutsui presented similar results at the Annual Meeting of the American Academy of Orthopaedic Surgeons.

Other Japanese surgeons have reported less satisfactory results. Masuda et al (1988), using Sugioka's technique, reported $69 \%$ satisfactory results in 52 patients followed for five years. Saito, Ohzono and Ono (1988) reported 15 patients with a follow-up of 2 to 14 years. They found absence of further collapse of the femoral head in nine patients, but had three fractures of the femoral neck and two cases of late varus deformity, a complication rate of $33 \%$.

Eyb and Kotz (1987) published the results of 39 Sugioka procedures with 23 good or excellent results, but they had a $46 \%$ incidence of postoperative complications which they related to the fixation device. We fixed all our osteotomies with a screw-plate because of concern with the poor fixation we achieved in preliminary work in the cadaver with the crossed wood screws proposed by Sugioka. We had no cases of malunion or nonunion.

Tooke, Amstutz and Hedley (1987) reported 17 patients at a mean follow-up of 39 months; seven (41\%) had had revision to hip replacement, three had virtual ankylosis, and three patients were dissatisfied. Only four patients $(24 \%)$ had excellent clinical results and one of these showed further radiographic collapse of the femoral head. These results, after a shorter follow-up, are similar to ours. The results of ${ }^{99 \mathrm{~m}} \mathrm{Tc}$ bone scanning in our study indicate that the remaining vascular supply of the femoral head is reduced by the operation.

There are several possible reasons for the striking differences between our results and those of Sugioka. First, we did not advise avoidance of weight-bearing after the osteotomies had healed; Sugioka's postoperative regimen apparently included a prolonged period without weight-bearing. Secondly, many of our osteotomies ended in a valgus rather than a varus position, but this did not seem to make any difference to the result.

The third and potentially most important reason may be that there are race-dependent differences in the anatomy of the hip capsule. In all our cases at osteotomy, we observed that anterior rotation of the femoral head stretched the quadratus femoris. The posterior collum branch of the medial circumflex artery which lies within this muscle may have become flattened and obstructed. It is possible that in Japanese patients, the posterior capsule of the hip is more lax and allows anterior rotation of the neck without compromising flow through this artery.

We abandoned the Sugioka anterior rotational osteotomy in 1983 and believe that it should not be performed in Caucasian patients. It does give satisfactory early pain relief, perhaps through joint denervation produced by the capsulotomy, but the procedure appears to compromise further the blood supply of the femoral head and to increase both collapse of the femoral head and secondary degenerative changes.

We found that the Sugioka procedure did not affect the results of subsequent total hip arthroplasty, partly because we performed the osteotomy with particular care to preserve the anatomy of the intertrochanteric area of the femur.

No benefits in any form have been received or will be received from a commercial party related directly or indirectly to the subject of this article.

\section{REFERENCES}

Eyb R, Kotz R. The transtrochanteric anterior rotational osteotomy of Sugioka: early and late results in idiopathic aseptic femoral head necrosis. Arch Orthop Trauma Surg 1987; 106:161-7.

Ficat RP, Arlet J. Ischemia and necrosis of bone. Baltimore, etc: Williams \& Wilkins, 1980:53-74.

Masuda T, Matsuno T, Hasegawa I, et al. Results of transtrochanteric rotational osteotomy for nontraumatic osteonecrosis of the femoral head. Clin Orthop 1988; $228: 69-74$.

Merle D'Aubigné R, Postel M, Mazabrand A, Mascias P, Gueguen J. Idiopathic necrosis of the femoral head in adults. J Bone Joint Surg [Br] 1965; 47-B:612-33.

Saito S, Ohzono K, Ono K. Joint-preserving operations for idiopathic avascular necrosis of the femoral head. J Bone Joint Surg [Br] $1988 ; 70-\mathrm{B}: 78-84$.

Sugioka Y. Transtrochanteric anterior rotational osteotomy of the femoral head in the treatment of osteonecrosis affecting the hip: a new osteotomy operation. Clin Orthop 1978; 130:191-201.

Sugioka Y. Transtrochanteric rotational osteotomy in the treatment of idiopathic and steroid-induced femoral head necrosis, Perthes' disease, slipped capital femoral epiphysis, and osteoarthritis of the hip: indications and results. Clin Orthop 1984; 184; 12-23.

Sugioka Y, Katsukj I, Hotokebuchi T. Transtrochanteric rotational osteotomy of the femoral head for the treatment of osteonecrosis: follow-up statistics. Clin Orthop 1982; 169:115-26.

Sugjoka Y, Hotokebuchi T, Tsutsui H. Long term follow-up study of transtrochanteric rotational osteotomy for idiopathic and steroidinduced femoral head necrosis. Trans 58th Annual Meeting of the AAOS, Anaheim, 1991.

Tooke SMT, Amstutz HC, Hedley AK. Results of transtrochanteric rotational osteotomy for femoral head osteonecrosis. Clin Orthop $1987 ; 224: 150-7$. 